\title{
Global Robust Attractive and Invariant Sets of Fuzzy Neural Networks with Delays and Impulses
}

\author{
Kaihong Zhao, ${ }^{1}$ Liwenjing Wang, ${ }^{1}$ and Juqing Liu ${ }^{2}$ \\ ${ }^{1}$ Center of Engineering Mathematics and Department of Applied Mathematics, Kunming University of Science and Technology, \\ Kunming, Yunnan 650093, China \\ ${ }^{2}$ Department of Mathematics, Yuxi Normal University, Yuxi, Yunnan 653100, China
}

Correspondence should be addressed to Kaihong Zhao; zhaokaihongs@126.com

Received 15 October 2012; Accepted 21 January 2013

Academic Editor: Huijun Gao

Copyright (C) 2013 Kaihong Zhao et al. This is an open access article distributed under the Creative Commons Attribution License, which permits unrestricted use, distribution, and reproduction in any medium, provided the original work is properly cited.

\begin{abstract}
A class of fuzzy neural networks (FNNs) with time-varying delays and impulses is investigated. With removing some restrictions on the amplification functions, a new differential inequality is established, which improves previouse criteria. Applying this differential inequality, a series of new and useful criteria are obtained to ensure the existence of global robust attracting and invariant sets for FNNs with time-varying delays and impulses. Our main results allow much broader application for fuzzy and impulsive neural networks with or without delays. An example is given to illustrate the effectiveness of our results.
\end{abstract}

\section{Introduction}

The theoretical and applied studies of the current neural networks (CNNs) have been a new focus of studies worldwide because CNNs are widely applied in signal processing, image processing, pattern recognition, psychophysics, speech, perception, robotics, and so on. The scholars have introduced many classes of CNNs models such as Hopfield-type networks [1], bidirectional associative memory networks [2], cellular neural networks [3], recurrent back-propagation networks [4-6], optimization-type networks [7-9], brain-statein-a-box-(BSB-) type networks [10,11], and Cohen-Grossberg recurrent neural networks (CGCNNs) [12]. According to the choice of the variable for CNNs [13], two basic mathematical models of CNNs are commonly adopted: either local field neural network models or static neural network models. The basic model of local field neural network is described as

$$
\dot{x}_{i}(t)=-x_{i}(t)+\sum_{j=1}^{n} \omega_{i j} f_{i}\left(x_{j}(t)\right)+I_{i}, \quad i=1,2, \ldots, n,
$$

where $f_{j}$ denotes the activation function of the $j$ th neuron; $x_{i}$ is the state of the $i$ th neuron; $I_{i}$ is the external input imposed on the $i$ th neuron; $\omega_{i j}$ denotes the synaptic connectivity value between the $i$ th neuron and the $j$ th neuron; $n$ is the number of neurons in the network.

It is well known that local field neural network not only models Hopfield-type networks but also models bidirectional associative memory networks and cellular neural networks. In the past few years, there has been increasing interest in studying dynamical characteristics such as stability, persistence, periodicity, robust stability of equilibrium points, and domains of attraction of local field neural network. Many deep theoretical results have been obtained for local field neural network. We can refer to [14-32] and the references cited therein.

However, in mathematical modeling of real world problems, we will encounter some other inconveniences, for example, the complexity and the uncertainty or vagueness. Fuzzy theory is considered as a more suitable setting for the sake of taking vagueness into consideration. Based on traditional cellular neural networks (CNNs),T. Yang and L.B. Yang proposed the fuzzy CNNs (FCNNs) [33], which integrate fuzzy logic into the structure of traditional CNNs and maintain local connectedness among cells. Unlike previous CNNs structures, FCNNs have fuzzy logic between its 
template input and/or output besides the sum of product operation. FCNNs are very useful paradigm for image processing problems, which is a cornerstone in image processing and pattern recognition. In addition, many evolutionary processes in nature are characterized by the fact that their states are subject to sudden changes at certain moments and therefore can be described by impulsive system. Therefore, it is necessary to consider both the fuzzy logic and delay effect on dynamical behaviors of neural networks with impulses. Nevertheless, to the best of our knowledge, there are few published papers considering the global robust domain of attraction for the fuzzy neural network (FNNs). Therefore, in this paper, we will study the global robust attracting set and invariant set of the following fuzzy neural networks (FNNs) with time-varying delays and impulses:

$$
\begin{aligned}
\dot{u}_{i}(t)= & -c_{i}(\lambda) u_{i}(t)+\sum_{j=1}^{n} \omega_{i j}(\lambda) f_{j}\left(u_{j}\left(t-\tau_{i j}(t)\right)\right) \\
& +\sum_{j=1}^{n} \gamma_{i j}(\lambda) v_{j}+I_{i}+\bigwedge_{j=1}^{n} a_{i j}(\lambda) f_{j}\left(u_{j}(t)\right) \\
& +\bigwedge_{j=1}^{n} \alpha_{i j}(\lambda) f_{j}\left(u_{j}\left(t-\tau_{i j}(t)\right)\right)+\bigwedge_{j=1}^{n} c_{i j}(\lambda) v_{j} \\
& +\bigvee_{j=1}^{n} b_{i j}(\lambda) f_{j}\left(u_{j}(t)\right)+\bigvee_{j=1}^{n} \beta_{i j}(\lambda) f_{j}\left(u_{j}\left(t-\tau_{i j}(t)\right)\right) \\
& +\bigvee_{j=1}^{n} \delta_{i j}(\lambda) v_{j}, \quad t \neq t_{k}, t \geq 0, \\
\Delta u_{i}(t)= & u_{i}\left(t^{+}\right)-u_{i}\left(t^{-}\right)=\mu_{i k}(\lambda) u_{i}(t), \quad t=t_{k}, t \geq 0, \\
& u_{i}(t)=\phi_{i}(t), \quad t \in[-\tau(\lambda), 0],
\end{aligned}
$$

where $\gamma_{i j}(\lambda)$ are elements of fuzzy feed-forward template, $a_{i j}(\lambda)$ and $\alpha_{i j}(\lambda)$ are elements of fuzzy feedback MIN template, $b_{i j}(\lambda)$ and $\beta_{i j}(\lambda)$ are elements of fuzzy feedback MAX template, and $c_{i j}(\lambda)$ and $\delta_{i j}(\lambda)$ are elements of fuzzy feedforward MIN template and fuzzy feed-forward MAX template, respectively. $\omega_{i j}(\lambda)$ is the weight of connection between the $i$ th neurons and the $j$ th neurons. $u_{i}(t), I_{i}$, and $v_{i}$ stand for state, input, and bias of the $i$ th neurons, respectively. $\tau_{i j}(t)$ is the transmission delay and $f_{j}$ is the activation function. $\wedge$ and $\vee$ denote the fuzzy AND and fuzzy OR operation, respectively. $\Delta u_{i}\left(t_{k}\right)$ is the impulses at moments $t_{k}$, and $0 \leq$ $t_{1}<t_{2}<\cdots$ is a strictly increasing sequence such that $\lim _{k \rightarrow \infty} t_{k}=+\infty . g_{i}=\mu_{i k}(\lambda) u_{i}(t)$ is the impulsive function. Function $\phi_{i}$ is the initial function. $\tau(\lambda)>0$ is a constant. $\lambda \in \Xi \subset R$ is the parameter.

The main purpose of this paper is to investigate the global robust attracting and invariant sets of FNNs (2). Different from $[34,35]$, in this paper, we will introduce a new nonlinear differential inequality, which is more effective than the linear differential inequalities for studying the asymptotic behavior of some nonlinear differential equations. Applying this new nonlinear delay differential inequality, sufficient conditions are gained for global robust attracting and invariant sets.

The rest of this paper is organized as follows. In Section 2, we will give some basic definitions and basic results about the attracting domains of FNNs (2). In Section 3, we will obtain the proof of the usefully nonlinear delay differential inequality. In Section 4, our main results will be proved by this delay differential inequality. Finally, an example is given to illustrate the effectiveness of our results in Section 5.

\section{Preliminaries}

As usual, $C(X, Y)$ denotes the space of continuous mappings from the topological space $X$ to the topological space $Y$. In particular, let $C\left([-\tau(\lambda), 0], \mathbb{R}^{n}\right)$ denote the set of all realvalued continuous mappings from $[-\tau(\lambda), 0]$ to $\mathbb{R}^{n}$ equipped with supremum norm $\|\cdot\|_{\infty}$ defined by

$$
\|\phi\|=\max _{1 \leq i \leq n} \sup _{-\tau(\lambda)<t \leq 0}\left|\phi_{i}(t)\right|
$$

where $\phi=\left(\phi_{1}, \phi_{2}, \ldots, \phi_{n}\right)^{T} \in C\left([-\tau(\lambda), 0], \mathbb{R}^{n}\right)$. Denote by $u(t, \phi, \lambda)$ the solution of FCNNs (2) with initial condition $\phi \in$ $C\left([-\tau(\lambda), 0], \mathbb{R}^{n}\right)$.

Let $E$ denote the $n$-dimensional unit matrix. For $A, B \in$ $\mathbb{R}^{m \times n}$ or $A, B \in \mathbb{R}^{n}, A \geq B(A>B)$ means that each pair of the corresponding elements of $A$ and $B$ satisfies the inequality “ $\geq(>)$ ". For any $x \in \mathbb{R}^{n}, A \in \mathbb{R}^{n \times n}, \varphi \in C\left([-\tau, 0], \mathbb{R}^{n}\right)$, we define $[x]^{+}=\left(\left|x_{1}\right|,\left|x_{2}\right|, \ldots,\left|x_{n}\right|\right)^{T}=\operatorname{col}\left\{\left|x_{i}\right|\right\},[A]^{+}=$ $\left(\left|a_{i j}\right|\right)_{n \times n},[\varphi(t)]_{\tau}=\left(\left[\varphi_{1}(t)\right]_{\tau},\left[\varphi_{2}(t)\right]_{\tau}, \ldots,\left[\varphi_{n}(t)\right]_{\tau}\right)^{T},[\varphi(t)]_{\tau}^{+}=$ $\left[[\varphi(t)]^{+}\right]_{\tau},\left[\varphi_{i}(t)\right]_{\tau}=\sup _{-\tau \leq \theta \leq 0}\left\{\varphi_{i}(t+\theta)\right\}$. For an $M$-matrix $D$ [36], we denote $D \in \mathscr{M}$ and $\Omega_{M}(D)=\left\{z \in R^{n}\right.$ : $D z>0, z>0\}$. For the sake of simplicity, we denote that $\bar{g}=\sup _{\lambda \in \Xi} \mathcal{G}(\lambda), \underline{g}=\inf _{\lambda \in \Xi} \mathcal{g}(\lambda)$, where $g(\lambda)$ is bounded in $\Xi$.

As usual, in the theory of impulsive differential equations, at the points of discontinuity $t_{k}, k=1,2, \ldots$, we assume that $u_{i}\left(t_{k}\right) \equiv u_{i}\left(t_{k}^{-}\right)$and $u_{i}^{\prime}\left(t_{k}\right) \equiv u_{i}^{\prime}\left(t_{k}^{-}\right)$.

Inspired by [37], we construct an equivalent theorem between (2) and (4). Then we establish some lemmas which are necessary in the proof of the main results.

Throughout this paper, we always assume the following.

$\left(A_{1}\right)$ For all $\lambda \in \Xi, 0<\left|\mu_{i k}(\lambda)\right|<1$ and $\sum_{k=1}^{\infty} \mu_{i k}(\lambda)$ is uniformly absolute convergence.

$\left(A_{2}\right) c_{i}(\lambda)>0, \omega_{i j}(\lambda), \gamma_{i j}(\lambda), a_{i j}(\lambda), b_{i j}(\lambda), c_{i j}(\lambda), \alpha_{i j}(\lambda)$, $\beta_{i j}(\lambda)$, and $\delta_{i j}(\lambda)$ are bounded in $\Xi(i, j=1,2, \ldots, n)$.

$\left(A_{3}\right)$ The activation function $f_{i}(\cdot)$ with $f_{i}(0)=0, i=$ $1,2, \ldots, n$ is second-order differentiable and Lipschitz continuous; that is, there exist positive constants $L_{i}$ such that $\left|f_{i}(x)\right| \leq L_{i}|x|$ for any $x \in \mathbb{R}$.

$\left(A_{4}\right)$ Functions $\tau_{i j}(t), i, j=1,2, \ldots, n$ are nonnegative, bounded, and continuous defined on $\mathbb{R}^{+}$and $0 \leq$ $\tau_{i j}(t) \leq \tau(\lambda)$. 
$\left(A_{5}\right)$ Let $\widehat{D}=-(\widehat{P}+\widehat{Q}) \in \mathscr{M}$, where $\widehat{P}=\left(\widehat{p}_{i j}\right)_{n \times n}, \widehat{p}_{i i}=$ $-\underline{c_{i}}+\left(\left|\bar{a}_{i i}\right|+\left|\bar{b}_{i i}\right|\right) L_{i}, \widehat{p}_{i j}=L_{i} \prod_{k=1}^{\infty}\left(1-\left|\bar{\mu}_{i k}\right|\right)^{-1} \sum_{j=1, j \neq i}^{n}$ $\prod_{k=1}^{\infty}\left(1+\left|\bar{\mu}_{j k}\right|\right)\left(\left|\bar{a}_{i j}\right|+\left|\bar{b}_{i j}\right|\right), \widehat{Q}=\left(\widehat{q}_{i j}\right)_{n \times n}, \widehat{q}_{i j}=$ $L_{i} \prod_{k=1}^{\infty}\left(1-\left|\bar{\mu}_{i k}\right|\right)^{-1} \sum_{j=1}^{n} \prod_{k=1}^{\infty}\left(1+\left|\bar{\mu}_{j k}\right|\right)\left(\left|\bar{\omega}_{i j}\right|+\left|\bar{\alpha}_{i j}\right|+\right.$ $\left.\left|\bar{\beta}_{i j}\right|\right)$, and $\widehat{I}=\operatorname{col}\left\{\left|I_{i}\right|+\sum_{j=1}^{n}\left(\left|\bar{\gamma}_{i j}\right|+\left|\bar{c}_{i j}\right|+\left|\bar{\delta}_{i j}\right|\right)\left|v_{j}\right|\right\}$.

Consider the following non-impulsive system (4):

$$
\begin{aligned}
\dot{x}_{i}(t)=- & c_{i}(\lambda) x_{i}(t)+\prod_{0 \leq t_{k}<t}\left(1+\mu_{i k}\right)^{-1} \\
\times & {\left[\sum_{j=1}^{n} \omega_{i j}(\lambda) f_{j}\left(\prod_{0 \leq t_{k}<t}\left(1+\mu_{j k}\right) x_{j}\left(t-\tau_{i j}(t)\right)\right)\right.} \\
& +\bigwedge_{j=1}^{n} a_{i j}(\lambda) f_{j}\left(\prod_{0 \leq t_{k}<t}\left(1+\mu_{j k}\right) x_{j}(t)\right) \\
& +\bigvee_{j=1}^{n} b_{i j}(\lambda) f_{j}\left(\prod_{0 \leq t_{k}<t}\left(1+\mu_{j k}\right) x_{j}(t)\right) \\
& +\bigwedge_{j=1}^{n} \alpha_{i j}(\lambda) f_{j}\left(\prod_{0 \leq t_{k}<t}\left(1+\mu_{j k}\right) x_{j}\left(t-\tau_{i j}(t)\right)\right) \\
& +\sum_{j=1}^{n} \gamma_{i j}(\lambda) v_{j}+\bigwedge_{j=1}^{n} c_{i j}(\lambda) v_{j} \\
& +\bigvee_{j=1}^{n} \delta_{i j}(\lambda) \gamma_{j}+I_{i j}(\lambda) f_{j}\left(\prod_{0 \leq t_{k}<t}\left(1+\mu_{j k}\right) x_{j}\left(t-\tau_{i j}(t)\right)\right) \\
& \prod_{j=1}(t)
\end{aligned}
$$$$
x_{i}(t)=\phi_{i}(t), \quad t \in[-\tau(\lambda), 0] .
$$

We have the following lemma, which shows that system (2) and (4) is equivalent.

Lemma 1. Assume $\left(A_{1}\right)$ holds, thenwe have the following.

(i) If $x_{i}(t)$ is a solution of (4), then $u_{i}(t)=\prod_{0 \leq t_{k}<t}(1+$ $\left.\mu_{i k}\right) x_{i}(t)$ is a solution of (2).

(ii) If $u_{i}(t)$ is a solution of (2), then $x_{i}(t)=\prod_{0 \leq t_{k}<t}(1+$ $\left.\mu_{i k}\right)^{-1} u_{i}(t)$ is a solution of (4).

Proof. Firstly, let us prove (i). For a given $\lambda \in \Xi$, it is easy to see that $u_{i}(t)=\prod_{0 \leq t_{k}<t}\left(1+\mu_{i k}(\lambda)\right) x_{i}(t)$ is absolutely continuous on the interval $\left(t_{k}, t_{k+1}\right]$ and for any $t \neq t_{k}, k=1,2, \ldots$,

$$
\begin{gathered}
u(t)=\left(\prod_{0 \leq t_{k}<t}\left(1+\mu_{1 k}(\lambda)\right) x_{1}(t), \ldots,\right. \\
\left.\prod_{0 \leq t_{k}<t}\left(1+\mu_{n k}(\lambda)\right) x_{n}(t)\right)
\end{gathered}
$$

satisfies system (2). In addition, for every $t_{k} \in\left\{t_{k}\right\}_{k=1}^{\infty}$,

$$
\begin{aligned}
& u_{i}\left(t_{k}^{+}\right)=\lim _{t \rightarrow t_{k}^{+}} \prod_{0 \leq t_{j}<t}\left(1+\mu_{i j}(\lambda)\right) x_{i}(t) \\
& =\prod_{0 \leq t_{j} \leq t_{k}}\left(1+\mu_{i j}(\lambda)\right) x_{i}(t) \\
& u_{i}\left(t_{k}\right)=\prod_{0 \leq t_{j}<t_{k}}\left(1+\mu_{i j}(\lambda)\right) x_{i}(t) .
\end{aligned}
$$

Thus, for every $k=1,2, \ldots$,

$$
u_{i}\left(t_{k}^{+}\right)=\left(1+\mu_{i k}(\lambda)\right) u_{i}\left(t_{k}\right) .
$$

The proof is complete.

Next, we prove (ii). Since $u_{i}(t)=\prod_{0 \leq t_{k}<t}\left(1+\mu_{i k}(\lambda)\right) x_{i}(t)$ is absolutely continuous on the interval $\left(t_{k}, t_{k+1}\right]$ and, in view of (7), it follows that, for any $k=1,2, \ldots$,

$$
\begin{aligned}
x_{i}\left(t_{k}^{+}\right) & =\prod_{0 \leq t_{j} \leq t_{k}}\left(1+\mu_{i j}(\lambda)\right)^{-1} u_{i}\left(t_{k}^{+}\right) \\
& =\prod_{0 \leq t_{j}<t_{k}}\left(1+\mu_{i j}(\lambda)\right)^{-1} u_{i}\left(t_{k}\right)=x_{i}\left(t_{k}\right), \\
x_{i}\left(t_{k}^{-}\right) & =\prod_{0 \leq t_{j} \leq t_{k-1}}\left(1+\mu_{i j}(\lambda)\right)^{-1} u_{i}\left(t_{k}^{-}\right) \\
& =x_{i}\left(t_{k}\right), \quad i=1,2, \ldots,
\end{aligned}
$$

which implies that $x_{i}(t)$ is continuous on $[0, \infty)$. It is easy to prove that $x_{i}(t)$ is absolutely continuous on $[0, \infty)$. Now, one can easily check that

$$
\begin{gathered}
x(t)=\left(\prod_{0 \leq t_{k}<t}\left(1+\mu_{1 k}(\lambda)\right)^{-1} u_{1}(t), \ldots,\right. \\
\left.\prod_{0 \leq t_{k}<t}\left(1+\mu_{n k}(\lambda)\right)^{-1} u_{n}(t)\right)
\end{gathered}
$$

is the solution of (4). The proof is complete.

Definition 2. Let $S$ be subsets of $C\left([-\tau(\lambda), 0], \mathbb{R}^{n}\right) \triangleq C$ which is independent of the parameter $\lambda \in \Xi$ and let $u(t, \phi, \lambda)$ be a solution of FNNs (2) with $\phi \in C$.

(i) For any given $\lambda \in \Xi$, if for any initial value $\phi \in S$ implies that $u(t, \phi, \lambda) \in S$ for all $t \geq 0$, then $S$ is said to be a robust positive invariant set of system of FNNs (2).

(ii) For any given $\lambda \in \Xi$, if for any initial value $\phi \in S$, the solution $u(t, \phi, \lambda) \in S$ converges to $S$ as $t \rightarrow \infty$, that is, $\operatorname{dist}(u(t, \phi, \lambda), S) \rightarrow 0$ as $t \rightarrow \infty$, then $S$ is said to be a global robust attracting set of system of FNNs (2), where $\operatorname{dist}(\varphi, S)=\inf _{\psi \in S} \operatorname{dist}(\varphi, \psi)$, and $\operatorname{dist}(\varphi, \psi)=$ $\sup _{s \in[-\tau, 0]}|\varphi(s)-\psi(s)|$ for $\varphi \in C$.

For a class of differential equations with the term of fuzzy AND and fuzzy OR operation, there is the following useful inequality. 
Lemma 3 (see [33]). Let $u=\left(u_{1}, u_{2}, \ldots, u_{n}\right)^{T}$ and $v=\left(v_{1}\right.$, $\left.v_{2}, \ldots, v_{n}\right)^{T}$ be two states of (2), then we have

$$
\begin{aligned}
& \left|\bigwedge_{j=1}^{n} \alpha_{i j} f_{j}\left(u_{j}\right)-\bigwedge_{j=1}^{n} \alpha_{i j} f_{j}\left(v_{j}\right)\right| \leq \sum_{j=1}^{n}\left|\alpha_{i j}\right|\left|f_{j}\left(u_{j}\right)-f_{j}\left(v_{j}\right)\right|, \\
& \left|\bigvee_{j=1}^{n} \alpha_{i j} f_{j}\left(u_{j}\right)-\bigvee_{j=1}^{n} \alpha_{i j} f_{j}\left(v_{j}\right)\right| \leq \sum_{j=1}^{n}\left|\alpha_{i j}\right|\left|f_{j}\left(u_{j}\right)-f_{j}\left(v_{j}\right)\right| .
\end{aligned}
$$

\section{Nonlinear Delay Differential Inequality}

In this section, we will establish a new nonlinear delay differential inequality which will play the important role to prove our main results.

Lemma 4. Assume that $u(t) \in C\left(\left[t_{0}, \infty\right), \mathbb{R}^{n}\right)$ satisfies

$$
\begin{aligned}
& \frac{d^{+}}{d t}[u(t)]^{+} \leq P[u(t)]^{+}+Q[u(t)]_{\tau}^{+}+I, \quad t \geq t_{0}, \\
& u\left(t_{0}+\theta\right)=\varphi(\theta) \in C\left([-\tau, 0], \mathbb{R}^{n}\right), \quad \theta \in[-\tau, 0],
\end{aligned}
$$

where $P=\left(p_{i j}\right)_{n \times n}$ and $p_{i j} \geq 0$ for $i \neq j, Q=\left(q_{i j}\right)_{n \times n} \geq 0$, $I=\left(I_{1}, I_{2}, \ldots, I_{n}\right)^{T} \geq 0$. If $D=-(P+Q) \in M$ and $L=D^{-1} I$, then we have the following.

(i) For any constant $d \geq 1$, the solution $u(t)$ of (11) satisfies

$$
[u(t)]^{+} \leq d L, \quad t \geq t_{0},
$$

provided that $[\varphi]_{\tau}^{+} \leq d L$.

(ii) Consider that

$$
[u(t)]^{+} \leq z e^{-\kappa\left(t-t_{0}\right)}+L, \quad t \geq t_{0},
$$

provided that

$$
[u(t)]^{+} \leq z e^{-\kappa\left(t-t_{0}\right)}+L, \quad t \in\left[t_{0}-\tau, t_{0}\right],
$$

where $z=\operatorname{col}\left\{\left|z_{i}\right|\right\} \in \Omega_{M}(D)$ and the positive constant $\kappa$ is determined by the following inequality:

$$
\left[\kappa E+P+Q e^{\kappa \tau}\right]<0 .
$$

Proof. Since $D=-(P+Q) \in \mathscr{M}$, we have $D^{-1} \geq 0$. Let $\varepsilon=$ $D^{-1} \operatorname{col}\{1\} \epsilon(\epsilon>0$ small enough), then $\varepsilon>0$. In order to prove (12), we will first prove that

$$
[u(t)]^{+} \leq d L+\varepsilon=\operatorname{col}\left\{\bar{x}_{i}\right\}=\bar{x}, \quad \forall t \geq t_{0},
$$

for any given initial function $\varphi \in C\left(\left[t_{0}-\tau, t_{0}\right], \mathbb{R}^{n}\right)$ with $[\varphi]_{\tau}^{+} \leq d L$.

If (16) does not hold, then there exist $i \in\{1,2, \ldots, n\}$ and $t_{1}>t_{0}$ such that

$$
\begin{gathered}
\left|u_{i}\left(t_{1}\right)\right|=\bar{x}_{i}, \quad[u(t)]^{+} \leq \bar{x}, \quad \text { for } t \leq t_{1}, \\
\frac{d^{+}}{d t}\left|u_{i}\left(t_{1}\right)\right| \geq 0
\end{gathered}
$$

It follows from (11) and (17) that

$$
\begin{aligned}
\frac{d^{+}}{d t}\left[u\left(t_{1}\right)\right]^{+} & \leq P\left[u\left(t_{1}\right)\right]^{+}+Q\left[u\left(t_{1}\right)\right]_{\tau}^{+}+I \leq(P+Q) \bar{x}+I \\
& =-[d I+\operatorname{col}\{1\} \epsilon-I] \leq-\operatorname{col}\{1\} \epsilon<0,
\end{aligned}
$$

which contradicts the inequality (18). So (16) holds for all $t \geq$ $t_{0}$. Letting $\epsilon \rightarrow 0$ in (16), we have

$$
[u(t)]^{+} \leq d L, \quad t \geq t_{0} .
$$

The proof of part (i) is complete.

Since $L=D^{-1} I$, we have $(P+Q) L+I=0$. Then

$$
\sum_{j=1}^{n}\left[p_{i j}+q_{i j}\right] L_{j}+I_{i}=0, \quad i=1,2, \ldots, n .
$$

From (15), we can get

$$
\sum_{j=1}^{n}\left[p_{i j}+q_{i j} e^{\kappa \tau}\right] z_{j}<-\kappa z_{i}, \quad i=1,2, \ldots, n .
$$

In the following, we at first will prove that for any positive constant $\epsilon$,

$$
\begin{array}{r}
\left|u_{i}(t)\right| \leq(1+\epsilon)\left[z_{i} e^{-\kappa\left(t-t_{0}\right)}+L_{i}\right]=w_{i}(t), \\
t \geq t_{0}, \quad i=1,2, \ldots, n .
\end{array}
$$

We let

$$
\begin{gathered}
\wp=\left\{i \in\{1,2, \ldots, n\}:\left|u_{i}(t)\right|>w_{i}(t)\right. \\
\text { for some } \left.t \in\left[t_{0},+\infty\right)\right\}, \\
\vartheta_{i}=\inf \left\{t \in\left[t_{0},+\infty\right):\left|u_{i}(t)\right|>w_{i}(t), i \in \wp\right\} .
\end{gathered}
$$

If inequality (23) is not true, then $\wp$ is nonempty set and there must exist some integer $m \in \wp$ such that $\vartheta_{m}=\min _{i \in \wp} \vartheta_{i} \in$ $\left[t_{0},+\infty\right)$.

By $u_{m}(t) \in([t-0,+\infty), \mathbb{R})$ and the inequality (23), we can get

$$
\begin{gathered}
\vartheta_{m}>t_{0}, \\
\left|u_{m}\left(\vartheta_{m}\right)\right|=w_{m}\left(\vartheta_{m}\right), \\
\frac{d^{+}}{d t}\left|u_{m}\left(\vartheta_{m}\right)\right| \geq \dot{w}_{m}\left(\vartheta_{m}\right), \\
\left|u_{i}(t)\right| \leq w_{i}(t), \quad t \in\left[t_{0}-\tau, \vartheta_{m}\right], i=1,2, \ldots, n .
\end{gathered}
$$

By applying (11) and (21)-(26), we obtain

$$
\begin{aligned}
& \frac{d^{+}}{d t}\left|u_{m}\left(\vartheta_{m}\right)\right| \\
& \leq \sum_{j=1}^{n}(1+\epsilon) z_{j} e^{-\kappa\left(\vartheta_{m}-t_{0}\right)}\left[p_{m m j}+q_{m j} e^{\kappa \tau}\right]-\epsilon I_{m} \\
& \leq \sum_{j=1}^{n}\left[p_{m j}+q_{m j} e^{\kappa \tau}\right](1+\epsilon) z_{j} e^{-\kappa\left(\vartheta_{m}-t_{0}\right)} \\
& <-\kappa z_{m}(1+\epsilon) e^{-\kappa\left(\vartheta_{m}-t_{0}\right)}=\dot{w}_{m}\left(\vartheta_{m}\right),
\end{aligned}
$$


which contradicts the inequality in (25). Thus the inequality (23) holds. Therefore, letting $\epsilon \rightarrow 0$, we have (13). The proof is complete.

By the process of proof of Lemma 4 , we easily derive the following theorem

Theorem 5. Under the conditions of Lemma 4, then

$$
\lim _{t \rightarrow+\infty}[u(t)]^{+} \leq L .
$$

\section{Main Results}

In this section, we will state and prove our main results. The following lemma is very useful to prove Theorem 7.

Lemma 6. Assume that $0<\left|a_{k}\right|<1,(k=1,2, \ldots)$ and the series of number $\sum_{k=1}^{\infty} a_{k}$ is absolute convergence, then the infinite products $\prod_{k=1}^{\infty}\left(1-\left|a_{k}\right|\right)^{-1}, \prod_{k=1}^{\infty}\left(1+a_{k}\right)$ and $\prod_{k=1}^{\infty}\left(1+\left|a_{k}\right|\right)$ are convergent and $\prod_{k=1}^{\infty}\left(1-\left|a_{k}\right|\right)^{-1} \geq \prod_{k=1}^{\infty}(1+$ $\left.a_{k}\right)^{-1}, \prod_{k=1}^{\infty}\left(1+\left|a_{k}\right|\right) \geq \prod_{k=1}^{\infty}\left(1+a_{k}\right)$.

Proof. In fact, by the assumption $0<\left|a_{k}\right|<1,(k=1,2, \ldots)$, we have

$$
\begin{gathered}
\frac{1}{1-\left|a_{k}\right|}>1, \quad \frac{1}{1-\left|a_{k}\right|} \geq \frac{1}{1+a_{k}}>0, \\
1+\left|a_{k}\right|>1, \quad 1+\left|a_{k}\right| \geq 1+a_{k} \geq 1-\left|a_{k}\right|>0
\end{gathered}
$$

which imply that

$$
\begin{gathered}
\ln \left(1-\left|a_{k}\right|\right) \leq \ln \left(1+a_{k}\right) \leq \ln \left(1+\left|a_{k}\right|\right), \\
\ln \left(1-\left|a_{k}\right|\right)<0, \quad \ln \left(1+\left|a_{k}\right|\right)>0 .
\end{gathered}
$$

On the other hand, since $\sum_{k=1}^{\infty} a_{k}$ is absolute convergence, we derive that

$$
\lim _{k \rightarrow \infty} a_{k}=\lim _{k \rightarrow \infty}\left|a_{k}\right|=0 .
$$

Equations (30) and (31) give that

$$
\begin{gathered}
\lim _{k \rightarrow \infty} \frac{-\ln \left(1-\left|a_{k}\right|\right)}{\left|a_{k}\right|}=1, \\
\lim _{k \rightarrow \infty} \frac{\ln \left(1+a_{k}\right)}{\left|a_{k}\right|}=1, \\
\lim _{k \rightarrow \infty} \frac{\ln \left(1+\left|a_{k}\right|\right)}{\left|a_{k}\right|}=1 .
\end{gathered}
$$

According to (32) and considering that $\sum_{k=1}^{\infty} a_{k}$ is absolute convergence, we get that the series of positive number $-\sum_{k=1}^{\infty} \ln \left(1-\left|a_{k}\right|\right)=\sum_{k=1}^{\infty} \ln \left(1-\left|a_{k}\right|\right)^{-1}, \sum_{k=1}^{\infty} \ln (1+$ $\left.a_{k}\right)$, and $\sum_{k=1}^{\infty} \ln \left(1+\left|a_{k}\right|\right)$ is convergent. Thus the infinite products $\prod_{k=1}^{\infty}\left(1-\left|a_{k}\right|\right)^{-1}, \prod_{k=1}^{\infty}\left(1+a_{k}\right)$, and $\prod_{k=1}^{\infty}\left(1+\left|a_{k}\right|\right)$ are convergent. At the same time, combined with (29), we conclude that $\prod_{k=1}^{\infty}\left(1-\left|a_{k}\right|\right)^{-1} \geq \prod_{k=1}^{\infty}\left(1+a_{k}\right)^{-1}, \prod_{k=1}^{\infty}(1+$ $\left.\left|a_{k}\right|\right) \geq \prod_{k=1}^{\infty}\left(1+a_{k}\right)$. The proof of Lemma 6 is complete.
Theorem 7. Assume that $\left(A_{1}\right)-\left(A_{5}\right)$ hold, then

$$
S=\left\{\phi \in C:[\phi]_{\tau(\lambda)}^{+} \leq \Pi \widehat{D}^{-1} \widehat{I}\right\}
$$

is a robust positive invariant and global robust attracting set of FNNs (2), where

$$
\Pi=\operatorname{diag}\left(\prod_{k=1}^{\infty}\left(1+\left|\bar{\mu}_{1 k}\right|\right), \prod_{k=1}^{\infty}\left(1+\left|\bar{\mu}_{2 k}\right|\right), \ldots, \prod_{k=1}^{\infty}\left(1+\left|\bar{\mu}_{n k}\right|\right) .\right.
$$

Proof. Calculating the upper right derivative $\left(d^{+} / d t\right)[x(t)]^{+}$ along system (4) and by using Lemma 6, we have

$$
\begin{aligned}
& \frac{d^{+}}{d t}\left|x_{i}(t)\right| \\
& =\operatorname{sgn}\left(x_{i}(t)\right) \dot{x}_{i}(t) \\
& \leq\left(-\underline{c}_{i}+L_{i}\left|\bar{a}_{i i}\right|+L_{i}\left|\bar{b}_{i i}\right|\right)\left|x_{i}(t)\right| \\
& +L_{i} \prod_{t_{k}<t}\left(1+\mu_{i k}\right)^{-1} \sum_{j=1, j \neq i}^{n} \prod_{t_{k}<t}\left(1+\mu_{j k}\right) \\
& \times\left(\left|\bar{a}_{i j}\right|+\left|\bar{b}_{i j}\right|\right)\left|x_{j}(t)\right| \\
& +L_{i} \prod_{t_{k}<t}\left(1+\mu_{i k}\right)^{-1} \sum_{j=1}^{n} \prod_{t_{k}<t}\left(1+\mu_{j k}\right) \\
& \times\left(\left|\bar{\omega}_{i j}\right|+\left|\bar{a}_{i j}\right|+\left|\bar{b}_{i j}\right|\right) \\
& \times\left|x_{j}\left(t-\tau_{i j}(t)\right)\right| \\
& +\left|I_{i}\right|+\sum_{j=1}^{n}\left(\left|\bar{\gamma}_{i j}\right|+\left|\bar{c}_{i j}\right|+\left|\bar{\delta}_{i j}\right|\right)\left|v_{j}\right| \\
& \leq\left(-\underline{c}_{i}+L_{i}\left|\bar{a}_{i i}\right|+L_{i}\left|\bar{b}_{i i}\right|\right)\left|x_{i}(t)\right| \\
& +L_{i} \prod_{t_{k}<t}\left(1-\left|\bar{\mu}_{i k}\right|\right)^{-1} \sum_{j=1, j \neq i}^{n} \prod_{t_{k}<t}\left(1+\left|\bar{\mu}_{j k}\right|\right) \\
& \times\left(\left|\bar{a}_{i j}\right|+\left|\bar{b}_{i j}\right|\right)\left|x_{j}(t)\right| \\
& +L_{i} \prod_{t_{k}<t}\left(1-\left|\bar{\mu}_{i k}\right|\right)^{-1} \sum_{j=1}^{n} \prod_{t_{k}<t}\left(1+\left|\bar{\mu}_{j k}\right|\right) \\
& \times\left(\left|\bar{\omega}_{i j}\right|+\left|\bar{a}_{i j}\right|+\left|\bar{b}_{i j}\right|\right) \\
& \times\left|x_{j}\left(t-\tau_{i j}(t)\right)\right| \\
& +\left|I_{i}\right|+\sum_{j=1}^{n}\left(\left|\bar{\gamma}_{i j}\right|+\left|\bar{c}_{i j}\right|+\left|\bar{\delta}_{i j}\right|\right)\left|v_{j}\right|
\end{aligned}
$$




$$
\begin{aligned}
& \leq\left(-\underline{c}_{i}+L_{i}\left|\bar{a}_{i i}\right|+L_{i}\left|\bar{b}_{i i}\right|\right)\left|x_{i}(t)\right| \\
& +L_{i} \prod_{k=1}^{\infty}\left(1-\left|\bar{\mu}_{i k}\right|\right)^{-1} \sum_{j=1, j \neq i}^{n} \prod_{k=1}^{\infty}\left(1+\left|\bar{\mu}_{j k}\right|\right) \\
& \quad \times\left(\left|\bar{a}_{i j}\right|+\left|\bar{b}_{i j}\right|\right)\left|x_{j}(t)\right| \\
& +L_{i} \prod_{k=1}^{\infty}\left(1-\left|\bar{\mu}_{i k}\right|\right)^{-1} \sum_{j=1}^{n} \prod_{k=1}^{\infty}\left(1+\left|\bar{\mu}_{j k}\right|\right) \\
& \quad \times\left(\left|\bar{\omega}_{i j}\right|+\left|\bar{a}_{i j}\right|+\left|\bar{b}_{i j}\right|\right) \\
& \times\left|x_{j}\left(t-\tau_{i j}(t)\right)\right| \\
& +\left|I_{i}\right|+\sum_{j=1}^{n}\left(\left|\bar{\gamma}_{i j}\right|+\left|\bar{c}_{i j}\right|+\left|\bar{\delta}_{i j}\right|\right)\left|\nu_{j}\right| .
\end{aligned}
$$

From $\left(A_{5}\right),(35)$ can be rewritten as follows:

$$
\frac{d^{+}}{d t}[x(t)]^{+} \leq \widehat{P}[x(t)]^{+}+\widehat{Q}[x(t)]_{\tau(\lambda)}^{+}+\widehat{I}, \quad t \geq 0 .
$$

Then from the conclusion (i) of Lemma 4, we can obtain

$$
[x(t)]^{+} \leq \widehat{L}_{1}, \quad t \geq 0
$$

provided that $[\phi]_{\tau(\lambda)}^{+} \leq \widehat{L}_{1}$, where $\widehat{L}_{1}=\widehat{D}^{-1} \widehat{I}$.

According to Lemma 1 and (37), one has

$$
\begin{aligned}
& {[u(t)]^{+}} \\
& =\operatorname{diag}\left(\prod_{0 \leq t_{k}<t}\left(1+\mu_{1 k}\right), \ldots, \prod_{0 \leq t_{k}<t}\left(1+\mu_{n k}\right)\right)[x(t)]^{+} \\
& \leq \operatorname{diag}\left(\prod_{k=1}^{\infty}\left(1+\left|\bar{\mu}_{1 k}\right|\right), \ldots, \prod_{k=1}^{\infty}\left(1+\left|\bar{\mu}_{n k}\right|\right)\right)[x(t)]^{+} \\
& =\Pi[x(t)]^{+} \leq \Pi \widehat{L}_{1}=\widehat{L},
\end{aligned}
$$

provided that $[\phi]_{\tau(\lambda)}^{+} \leq \widehat{L}$, where $\widehat{L}=\Pi \widehat{D}^{-1} \widehat{I}$. In view of Definition 2, we get that $S$ denoted by (33) is a robust positive invariant set of FNNs (2).

On the other hand, since $\widehat{D} \in \mathscr{M}$, there exists a positive vector $z=\left(z_{1}, z_{2}, \ldots, z_{n}\right)^{T}$ such that

$$
\widehat{D} z>0, \quad \text { that is, }(\widehat{P}+\widehat{Q}) z<0 \text {. }
$$

By using continuity, we know that there must exist a positive scalar $\kappa(\lambda)$ such that

$$
\left[\kappa(\lambda) E+\widehat{P}+\widehat{Q} e^{\kappa(\lambda) \tau(\lambda)}\right] z<0
$$

where $\widehat{d} \geq 1$ is a constant such that $[\phi]_{\tau(\lambda)}^{+} \leq \widehat{d} \widehat{L}$.

Then by (36), (40), and $\left(A_{4}\right)$, all the conditions of Theorem 5 are satisfied, and we have

$$
\lim _{t \rightarrow+\infty}[x(t)]^{+} \leq \widehat{L}
$$

According to Lemma 1 and Definition 2, we yield that $S$ denoted by (33) is also a global robust attracting set of FNNs (2). The proof is complete.

Theorem 8. In addition to $\left(A_{1}\right)-\left(A_{5}\right)$, further assume $\widehat{I}=$ 0 . Then FNNs (2) has a zero solution and the zero solution is global robust exponential stability and the exponential convergent rate equals $\kappa$ which is determined by (40).

\section{Illustrative Example}

The following illustrative example will demonstrate the effectiveness of our results. Consider the following FNNs with time-varying delays and impulses:

$$
\begin{aligned}
\dot{u}_{i}(t)= & -c_{i}(\lambda) u_{i}(t)+\sum_{j=1}^{2} \omega_{i j}(\lambda) f_{j}\left(u_{j}\left(t-\tau_{i j}(t)\right)\right) \\
& +\sum_{j=1}^{n} \gamma_{i j}(\lambda) v_{j}+I_{i}+\bigwedge_{j=1}^{2} a_{i j}(\lambda) f_{j}\left(u_{j}(t)\right) \\
& +\bigwedge_{j=1}^{2} \alpha_{i j}(\lambda) f_{j}\left(u_{j}\left(t-\tau_{i j}(t)\right)\right)+\bigwedge_{j=1}^{n} c_{i j}(\lambda) v_{j} \\
& +\bigvee_{j=1}^{2} b_{i j}(\lambda) f_{j}\left(u_{j}(t)\right)+\bigvee_{j=1}^{2} \beta_{i j}(\lambda) f_{j}\left(u_{j}\left(t-\tau_{i j}(t)\right)\right) \\
& +\bigvee_{j=1}^{n} \delta_{i j}(\lambda) v_{j}, \quad t \neq t_{k}, t \geq 0, \\
\Delta u_{i}(t)= & u_{i}\left(t^{+}\right)-u_{i}\left(t^{-}\right)=\mu_{i k}(\lambda) u_{i}(t), \quad t=t_{k}, t \geq 0 \\
& u_{i}(t)=\phi_{i}(t), \quad t \in[-\tau(\lambda), 0]
\end{aligned}
$$

where $i=1,2, t_{k} \in\{1,2,3, \ldots\}, \Xi=[\pi / 4, \pi / 2], \tau(\lambda)=1$, $c_{1}(\lambda)=h(8+\sin (2 \lambda)), c_{2}(\lambda)=h(9+\cos (2 \lambda)), f_{1}(x)=f_{2}(x)=$ $(1 / 2)(|x+1|-|x-1|), \tau_{i j}(t)=|\sin (i-j) t|, \mu_{1 k}(\lambda)=\mu_{2 k}(\lambda)=$ $(-1)^{k} \sin \lambda / 2^{k}, a_{i i}(\lambda)=h(1+\sin 2 i \lambda) / 4, a_{i j}(\lambda)=((1+\sin (i+$ $j) \lambda) / 4)(i \neq j), b_{i i}(\lambda)=h(1+2 \sin (i+j) \lambda) / 6, b_{i j}(\lambda)=((1+$ $2 \sin (i+j) \lambda) / 6)(i \neq j), \alpha_{i j}(\lambda)=(2+\sin (i+j) \lambda) / 9, \beta_{i j}(\lambda)=$ $(1+2 \sin (i+j) \lambda) / 9, \omega_{i j}(\lambda)=(1+\sin (i+j) \lambda) / 6, c_{i j}(\lambda)=\sin (i+$ $j) \lambda / 4, \gamma_{i j}(\lambda)=-\sin (i+j) \lambda / 4$, and $\delta_{i j}(\lambda)=\cos (i+j) \lambda / 4, I_{i}=$ $-\left((-1)^{i} / 4\right), \nu_{i}=(-1)^{i}$. By the simple calculation, we obtain

$$
\widehat{P}=h\left(\begin{array}{cc}
-7 & 1 \\
1 & -7
\end{array}\right), \quad \widehat{Q}=h\left(\begin{array}{ll}
1 & 1 \\
1 & 1
\end{array}\right), \quad \widehat{I}=\left(\begin{array}{l}
1 \\
1
\end{array}\right),
$$

where $h=\prod_{k=1}^{\infty}\left(2^{k}+1\right) /\left(2^{k}-1\right)$. Then $\widehat{D}=-(\widehat{P}+\widehat{Q})$ is a nonsingular $M$-matrix, and taking $\kappa=0.1, z=(1,1)^{T}$, we get

$$
\left[\kappa E+\widehat{P}+\widehat{Q} e^{\kappa \tau}\right] z<0 .
$$

Therefore, by Theorem 7, we obtain that

$$
S=\left\{\phi \in C:[\phi]_{\tau(\lambda)}^{+} \leq \Pi \widehat{D}^{-1} \widehat{I}=\left(\frac{1}{4}, \frac{1}{4}\right)^{T}\right\}
$$


is a robust positive invariant and global robust attracting set of FNNs (42).

\section{Acknowledgments}

The author would like to thank the anonymous referees for their useful and valuable suggestions. This work is supported by the National Natural Sciences Foundation of China under Grant no. 11161025, Yunnan Province Natural Scientific Research Fund Project (no. 2011FZ058), and Yunnan Province Education Department Scientific Research Fund Project (no. 2011Z001).

\section{References}

[1] J. J. Hopfield, "Neurons with graded response have collective computational properties like those of two-state neurons," Proceedings of the National Academy of Sciences of the United States of America, vol. 81, no. 10, pp. 3088-3092, 1984.

[2] B. Kosko, "Bidirectional associative memories," IEEE Transactions on Systems, Man, and Cybernetics, vol. 18, no. 1, pp. 49-60, 1988.

[3] L. O. Chua and L. Yang, "Cellular neural networks: theory," IEEE Transactions on Circuits and Systems, vol. 35, no. 10, pp. 12571272, 1988.

[4] L.B. Almeida, "Backpropagation in perceptrons with feedback," in Neural Computers, pp. 199-208, Springer, New York, NY, USA, 1988.

[5] F. J. Pineda, "Generalization of back-propagation to recurrent neural networks," Physical Review Letters, vol. 59, no. 19, pp. 2229-2232, 1987.

[6] R. Rohwer and B. Forrest, "Training time-dependence in neural networks," in Proceedings of the 1st IEEE International Conference on Neural Networks, pp. 701-708, San Diego, Calif, USA, 1987.

[7] M. Forti and A. Tesi, "New conditions for global stability of neural networks with application to linear and quadratic programming problems," IEEE Transactions on Circuits and Systems. I, vol. 42, no. 7, pp. 354-366, 1995.

[8] Y. Xia and J. Wang, "A general methodology for designing globally convergent optimization neural networks," IEEE Transactions on Neural Networks, vol. 9, no. 6, pp. 1331-1343, 1998.

[9] Y. S. Xia and J. Wang, "On the stability of globally projected dynamical systems," Journal of Optimization Theory and Applications, vol. 106, no. 1, pp. 129-150, 2000.

[10] J.-H. Li, A. N. Michel, and W. Porod, "Analysis and synthesis of a class of neural networks: linear systems operating on a closed hypercube," IEEE Transactions on Circuits and Systems, vol. 36, no. 11, pp. 1405-1422, 1989.

[11] I. Varga, G. Elek, and S. H. Zak, "On the brain-state-in-aconvex-domain neural models," Neural Networks, vol. 9, no. 7, pp. 1173-1184, 1996.

[12] M. A. Cohen and S. Grossberg, "Absolute stability of global pattern formation and parallel memory storage by competitive neural networks," IEEE Transactions on Systems, Man, and Cybernetics, vol. 13, no. 5, pp. 815-826, 1983.

[13] H. Qiao, J. Peng, Z. B. Xu, and B. Zhang, "A reference model approach to stability analysis of neural networks," IEEE Transactions on Systems, Man, and Cybernetics, Part B, vol. 33, no. 6, pp. 925-936, 2003.
[14] X. Yang, X. Liao, Y. Tang, and D. J. Evans, "Guaranteed attractivity of equilibrium points in a class of delayed neural networks," International Journal of Bifurcation and Chaos in Applied Sciences and Engineering, vol. 16, no. 9, pp. 2737-2743, 2006.

[15] H. Zhao, "Global asymptotic stability of Hopfield neural network involving distributed delays," Neural Networks, vol. 17, no. 1, pp. 47-53, 2004.

[16] J. Cao and J. Wang, "Global asymptotic and robust stability of recurrent neural networks with time delays," IEEE Transactions on Circuits and Systems I, vol. 52, no. 2, pp. 417-426, 2005.

[17] Z. Huang, X. Wang, and F. Gao, "The existence and global attractivity of almost periodic sequence solution of discretetime neural networks," Physics Letters A, vol. 350, no. 3-4, pp. 182-191, 2006.

[18] Z. B. Xu, H. Qiao, J. Peng, and B. Zhang, "A comparative study of two modeling approaches in neural networks," Neural Networks, vol. 17, no. 1, pp. 73-85, 2004.

[19] M. Wang and L. Wang, "Global asymptotic robust stability of static neural network models with S-type distributed delays," Mathematical and Computer Modelling, vol. 44, no. 1-2, pp. 218222, 2006.

[20] P. Li and J. Cao, "Stability in static delayed neural networks: a nonlinear measure approach," Neurocomputing, vol. 69, no. 1315, pp. 1776-1781, 2006.

[21] Z. Huang and Y. Xia, "Exponential p-stability of second order Cohen-Grossberg neural networks with transmission delays and learning behavior," Simulation Modelling Practice and Theory, vol. 15, no. 6, pp. 622-634, 2007.

[22] K. Zhao and Y. Li, "Robust stability analysis of fuzzy neural network with delays," Mathematical Problems in Engineering, vol. 2009, Article ID 826908, 13 pages, 2009.

[23] R. Yang, H. Gao, and P. Shi, "Novel robust stability criteria for stochastic Hopfield neural networks with time delays," IEEE Transactions on Systems, Man, and Cybernetics, Part B, vol. 39, no. 2, pp. 467-474, 2009.

[24] R. Yang, Z. Zhang, and P. Shi, "Exponential stability on stochastic neural networks with discrete interval and distributed delays," IEEE Transactions on Neural Networks, vol. 21, no. 1, pp. 169-175, 2010.

[25] X. Li and J. Shen, "LMI approach for stationary oscillation of interval neural networks with discrete and distributed timevarying delays under impulsive perturbations," IEEE Transactions on Neural Networks, vol. 21, no. 10, pp. 1555-1563, 2010.

[26] Y. Zhao, L. Zhang, S. Shen, and H. Gao, "Robust stability criterion for discrete-time uncertain markovian jumping neural networks with defective statistics of modes transitions," IEEE Transactions on Neural Networks, vol. 22, no. 1, pp. 164-170, 2011.

[27] X. W. Li, H. J. Gao, and X. H. Yu, "A Unified Approach to the Stability of Generalized Static Neural Networks With Linear Fractional Uncertainties and Delays," IEEE Transactions on Systems, Man and Cybernetics, Part B, vol. 41, no. 5, pp. 12751286, 2011.

[28] H. Dong, Z. Wang, and H. Gao, "Robust $H_{\infty}$ filtering for a class of nonlinear networked systems with multiple stochastic communication delays and packet dropouts," IEEE Transactions on Signal Processing, vol. 58, no. 4, pp. 1957-1966, 2010.

[29] W. Zhang, Y. Tang, J.-A. Fang, and X. Wu, "Stability of delayed neural networks with time-varying impulses," Neural Networks, vol. 36, pp. 59-63, 2012. 
[30] H. Huang, T. Huang, and X. Chen, "Global exponential estimates of delayed stochastic neural networks with Markovian switching," Neural Networks, vol. 36, pp. 136-145, 2012.

[31] Q. T. Gan, "Exponential synchronization of stochastic CohenGrossberg neural networks with mixed time-varying delays and reactio-diffusion via periodically intermittent control," Neural Networks, vol. 31, pp. 12-21, 2012.

[32] Y. K. Li and C. Wang, "Existence and global exponential stability of equilibrium for discretetime fuzzy BAM neural networks with variable delays and impulses," Fuzzy Sets and Systems, 2012.

[33] T. Yang and L.-B. Yang, "The global stability of fuzzy cellular neural network," IEEE Transactions on Circuits and Systems. I, vol. 43 , no. 10 , pp. $880-883,1996$.

[34] D. Xu and Z. Yang, "Impulsive delay differential inequality and stability of neural networks," Journal of Mathematical Analysis and Applications, vol. 305, no. 1, pp. 107-120, 2005.

[35] Z. Yang and D. Xu, "Impulsive effects on stability of CohenGrossberg neural networks with variable delays," Applied Mathematics and Computation, vol. 177, no. 1, pp. 63-78, 2006.

[36] R. A. Horn and C. R. Johnson, Topics in Matrix Analysis, vol. 2, Cambridge University Press, Cambridge, UK, 1991.

[37] Y. Li, "Global exponential stability of BAM neural networks with delays and impulses," Chaos, Solitons and Fractals, vol. 24, no. 1, pp. 279-285, 2005. 


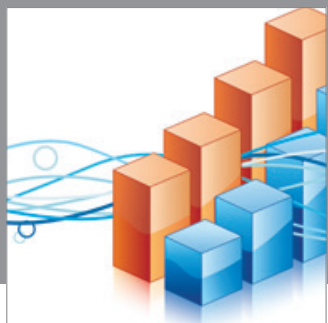

Advances in

Operations Research

mansans

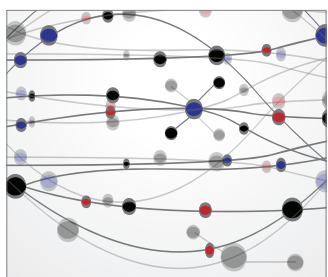

The Scientific World Journal
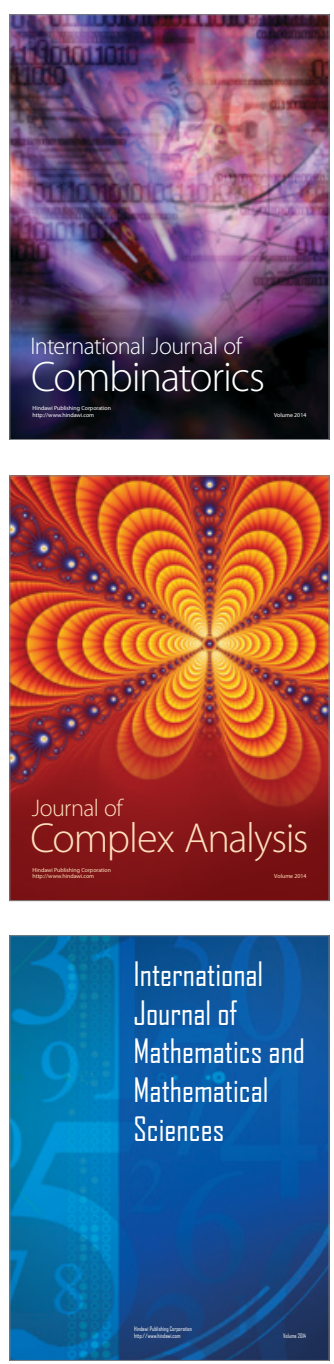
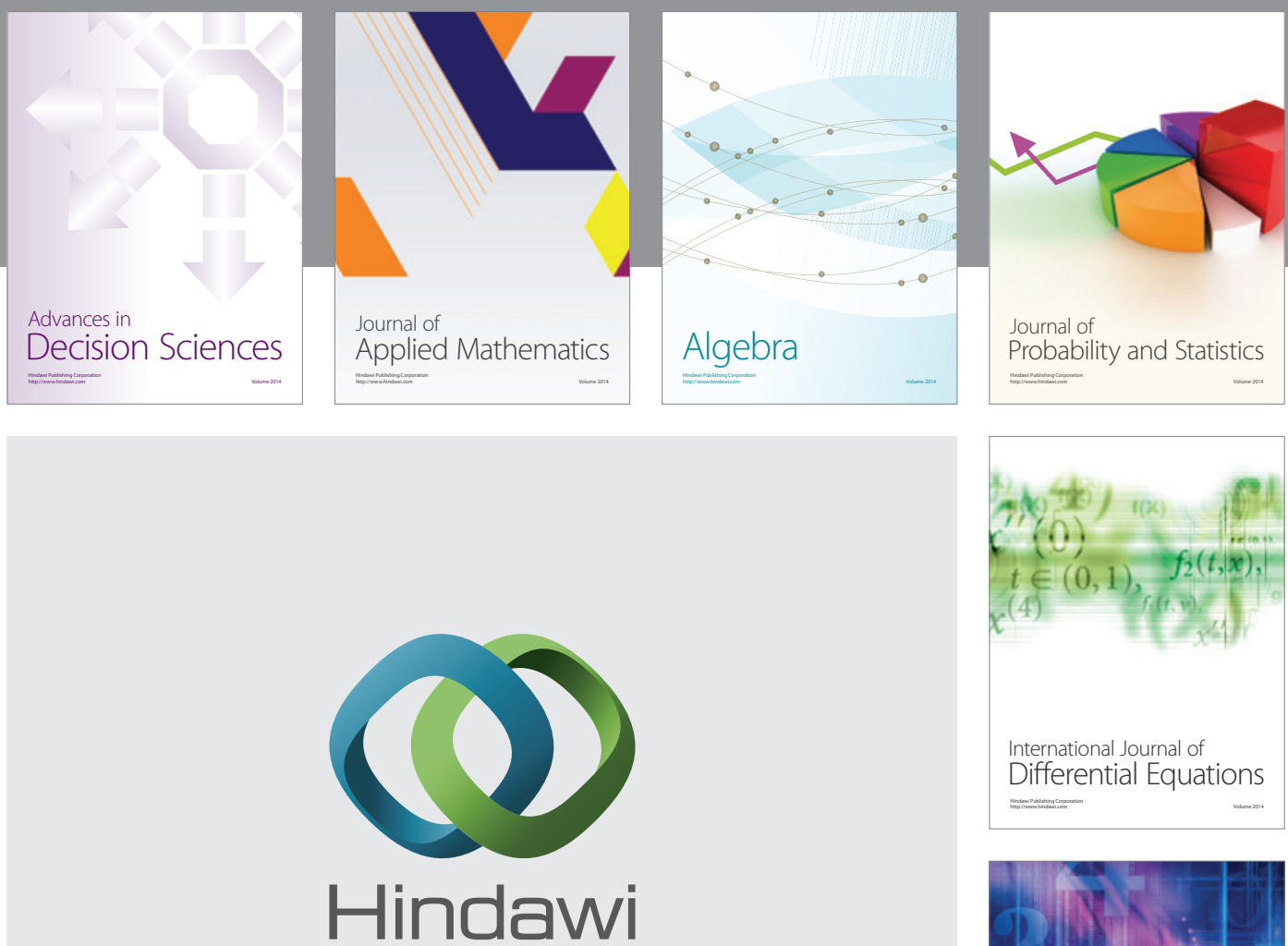

Submit your manuscripts at http://www.hindawi.com
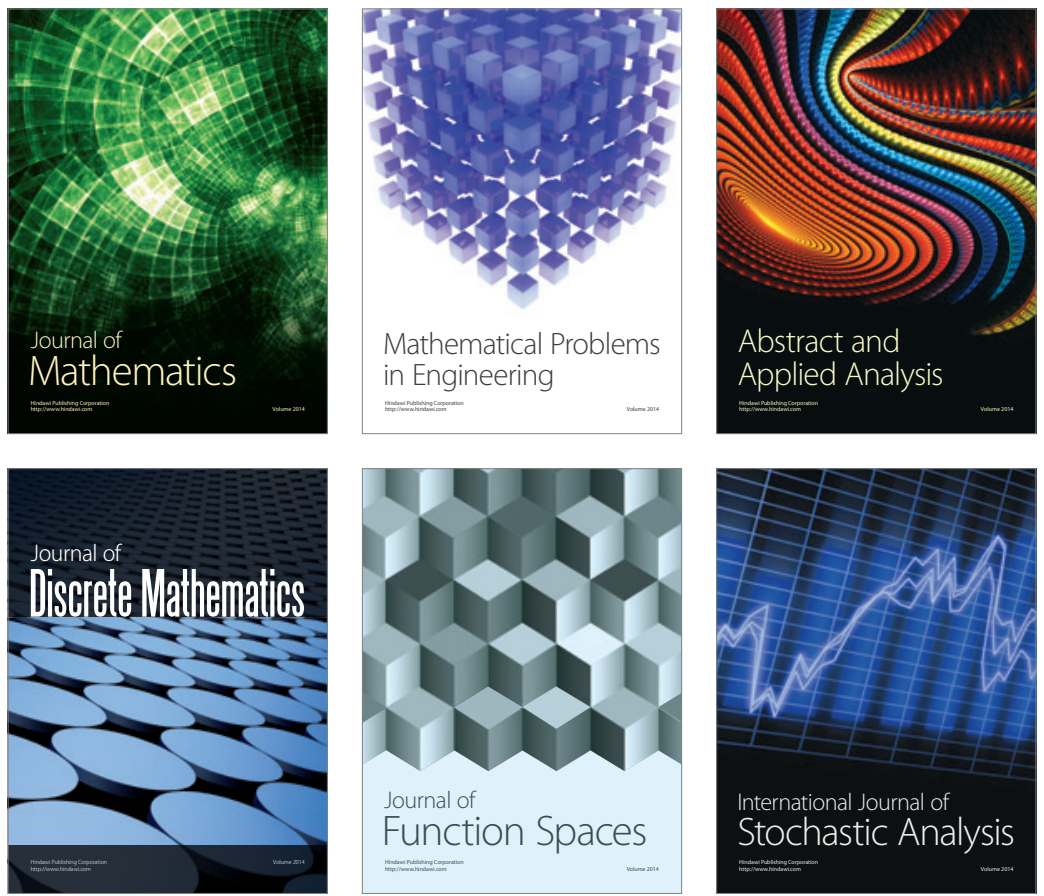

Journal of

Function Spaces

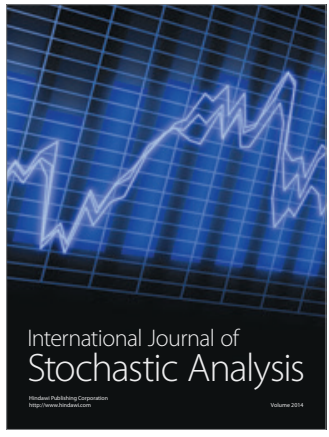

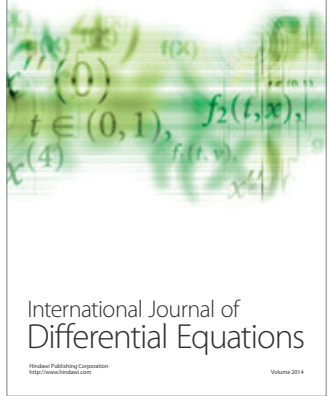
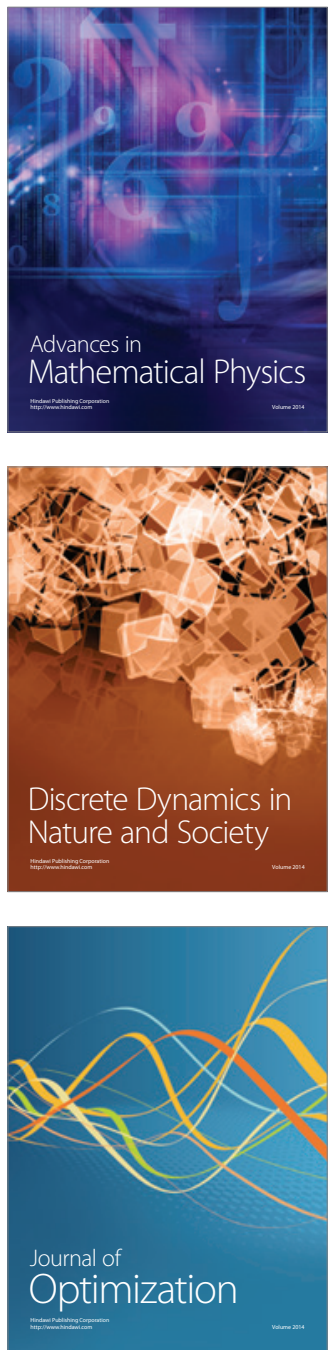